\title{
Correction to: Overview on cyanobacterial exopolysaccharides and biofilms: role in bioremediation
}

\author{
Akhilesh A. Potnis - Prashanth S. Raghavan - Hema Rajaram $\mathbb{C}$
}

Published online: 19 August 2021

(C) Springer Nature B.V. 2021

Correction to: Rev Environ Sci Biotechnol (2021) 20:781-794

https://doi.org/10.1007/s11157-02109586-w

The author names of "Nieboer" and "De Philippis" have been misspelled in the original publication of the article.
The correct version of author names are Nieboer and De Philippis respectively.

Authors have also apologized for the error.

Publisher's Note Springer Nature remains neutral with regard to jurisdictional claims in published maps and institutional affiliations.
The original article can be found online at https:// doi.org/10.1007/s11157-021-09586-w.

A. A. Potnis · P. S. Raghavan · H. Rajaram ( $\square)$ Molecular Biology Division, Bhabha Atomic Research Centre, Trombay, Mumbai 400085, India

e-mail: hemaraj@barc.gov.in

H. Rajaram

Homi Bhabha National Institute, Training School

Complex, Anushaktinagar, Mumbai 400094, India 